\section{Clinical v. laboratory-based screening for COVID-19 in asymptomatic patients requiring acute cardiac care}

To the Editor: Tygerberg Academic Hospital (TBH) and its network of satellite hospitals provide coronary care to 2.4 million people in and around Cape Town, South Africa. ${ }^{[1]}$ At the height of the COVID19 pandemic, weekly admissions to our coronary care unit (CCU) fell by $46 \%$ compared with the previous year, only returning to pre-COVID-19 levels after easing of the hard lockdown. ${ }^{[2]}$ This posthard lockdown period was particularly challenging, as COVID-19 admissions to TBH increased further, competing with higher numbers of CCU patients for limited bed space. To prevent nosocomial spread of COVID-19 in the CCU and protect staff working in this essential acute service, we aimed to maintain our $\mathrm{CCU}$ as a low-risk COVID-19 area. While nasopharyngeal swab polymerase chain reaction (PCR) remains the gold standard for diagnosing COVID-19 infection, its sensitivity for diagnosing acute infection is relatively low in symptomatic patients and its performance in asymptomatic patients remains largely unknown. ${ }^{[3,4]}$ Furthermore, it is unclear whether clinical screening without nasopharyngeal swab PCR is a sufficient strategy to minimise nosocomial transmission.

Our initial strategy was selective PCR testing. All patients referred to the Division of Cardiology at TBH were screened for symptoms of COVID-19 infection and underwent nasopharyngeal swab testing based on symptom profile. Patients with proven current COVID-19 infection and those in whom there was high clinical suspicion were admitted to a dedicated COVID-19 ward or single isolation rooms in the CCU pending their PCR swab results. Emergency interventions were performed using full PPE. ${ }^{[5]}$ During a 4-week period from 8 July to 5 August 2020, at the peak of the Cape Town pandemic, ${ }^{[6]}$ our selective testing strategy was changed to a strategy of testing all patients admitted to the CCU, including those without COVID-19 symptoms. The rationale for this change was the perception that there may be a significant number of asymptomatic COVID-19-positive patients at the height of the pandemic. Surveillance testing, as it was termed, did not influence patient management in any way.

During this period, 116 asymptomatic patients were admitted to the CCU; 89 surveillance swabs were performed (77\% of admissions), with similar numbers performed in the CCU (46\%) and at our referral hospitals (54\%)
Of the 89 surveillance swabs, 3 (3.4\%) were positive for COVID19. The swab-positive patients were isolated in single rooms in the CCU after the test result became available. Two patients underwent percutaneous coronary intervention, while the third underwent inpatient coronary bypass surgery after 10 days of isolation. None of these patients developed symptoms or adverse events during their admission, and all were discharged home, well. We did not see an increase in healthcare worker infections in the CCU, and there was no evidence of nosocomial spread to patients.

Our findings are reassuring for clinicians (and patients) attempting to return to normal practice. The number of asymptomatic patients with underlying COVID-19 infection admitted to hospital, even at the peak of the pandemic, appears to be low. Furthermore, our data are in keeping with recent reports that asymptomatic patients do not pose an increased risk for nosocomial spread of COVID-19, as long as basic COVID-19 care principles are adhered to. ${ }^{[7,8]}$

\section{H Joubert, P G Herbst, A F Doubell, A J K Pecoraro}

Division of Cardiology, Department of Internal Medicine, Faculty of Medicine and Health Sciences, Stellenbosch University and Tygerberg Hospital, Cape Town, South Africa

lloydjoubert@gmail.com; 14310139@sun.ac.za

1. Western Cape Government. Socio-economic profile: City of Cape Town 2017. 2017. https://www. westerncape govza/assets/departments/treasury/Documents/Socio-economic-profiles/2017/city_o_ 2010 . Pec Eur Heart J 2020 (in press).

Woolshin S, Patel N, Kesselheim AS. False negative tests for SARS-CoV-2 infection - challenges and implications. N Engl J Med 2020;38(1):1-2. https://doi.org/10.1056/NEJMp2009027

4. Shuren J, Stentzel T. Covid-19 molecular diagnostic testing - lessons learned. N Engl J Med 2020 (epub 9 September 2020). https://doi.org/10.1056/NEJMp2023830

5. John TJ, Hassan K, Weich H. Donning and doffing of personal protective equipment (PPE) fo angiography during the COVID-19 crisis. Eur Heart J 2020;41(19):1786-1787. https://doi.org/10.1093/ eurheartj/ehaa 283

6. Abdool Karim SS. The South African response to the pandemic. N Engl J Med 2020;382(24):1-3. https://doi.org/10.1056/NEJMc2014960

Meel R, Cupido BJ, Pecoraro AJK, Doubell AF, Lubbe W, Ntusi NAB. COVID-19 and cardiovascular imaging: A guide for the practising clinician. SA Heart 2019;17(3):302-308.

8. Gao M, Yang L, Chen X, et al. A study on infectivity of asymptomatic SARS-CoV-2 carriers. Respir Med 2020;169:106026. https://doi.org/10.1016/..rmed.2020.106026

S Afr Med J 2020;110(11):1064. https://doi.org/10.7196/SAMJ.2020.v110i11.15306 